\title{
Wrapping up memories
}

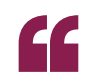

Together, these findings suggest that learninginduced adult oligodendrogenesis is important for memory consolidation

As myelin-producing oligodendrocytes are formed throughout the lifespan and oligodendrogenesis is regulated by neural activity, myelination patterns may change in an experiencedependent fashion, thereby representing a form of plasticity. Previous studies have indicated that myelin remodelling may have a role in memory encoding. Now, a new study finds that oligodendrogenesis is important for memory consolidation in adult mice.

To study the potential role of adult oligodendrogenesis in memory, the authors used mice in which the gene encoding myelin regulatory factor (MRF) - a transcription factor required by oligodendrocytes in order to myelinate - could be conditionally knocked out in oligodendrocyte precursor cells through tamoxifen (TAM) or 4-hydroxy-tamoxifen (4OHT) administration ( $M r f^{\mathrm{CKO}}$ mice). Young adult $M r f^{\mathrm{CKO}}$ mice showed reductions in adult-generated oligodendrocytes in the retrosplenial cortex (RSC) and the corpus callosum (CC) at 4 days after 4OHT treatment. Similar effects were observed in such mice 4 weeks after TAM administration, indicating that this method selectively targets adult oligodendrogenesis.

The authors next examined the effects of spatial learning on adult oligodendrocyte generation in hippocampal-cortical

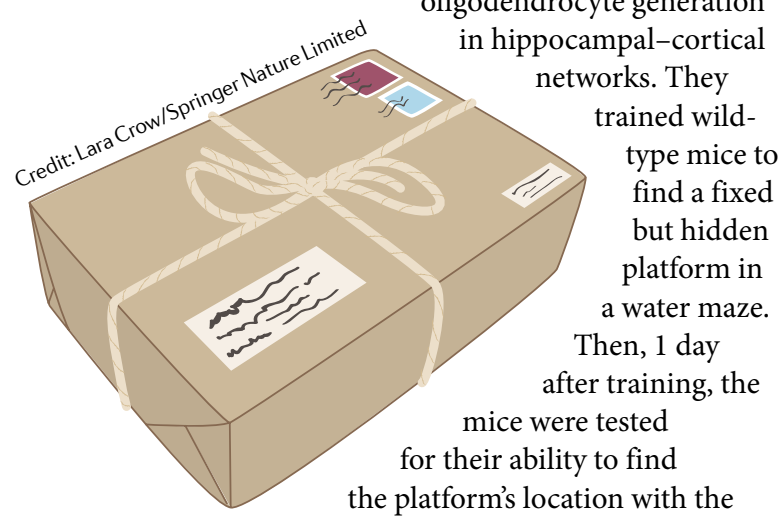

platform removed (the probe trial). As expected, the mice learned the task and this was accompanied by a rise in adult oligodendrocyte number in the anterior cingulate cortex (ACC), the RSC and the CA1 hippocampal region. In the same task, $M r f^{\text {CKO }}$ mice that received 4OHT during training did not perform as well as controls in the probe trial, suggesting that spatial learning is affected by impaired adult oligodendrogenesis.

The authors explored whether the observed adult oligodendrogenesis occurred during or after training. They administered to mice a thymidine analogue, to label newly generated cells, immediately after training in the platform task. Then, they assessed the spatial memory of the mice 1 day and 28 days after training and the incorporation of the analogue into cells at the latter time point. This approach showed that adult oligodendrogenesis increased during the post-training period in several of the brain regions assessed, including the ACC and the CC. Electron microscopy revealed an increase in the number of myelinated axons in the same regions following training, although there were no effects on myelin thickness. Together, these findings suggest that learning-induced adult oligodendrogenesis is important for memory consolidation and exerts its effects by inducing de novo myelination rather than affecting existing myelination.

To test the hypothesis that adult oligodendrogenesis is important for memory consolidation, the authors treated $M r f^{\mathrm{CKO}}$ mice with TAM immediately after training in the water maze and assessed these animals with probe trials 1 day and 28 days after training completion. In the first probe trial, the mice concentrated their search in the area where the platform had been located, but in the second trial, their searching was less selective, supporting the idea that adult oligodendrogenesis in the post-training period is necessary for memory consolidation. Further experiments revealed that impairment of spatial memory consolidation was linked to reductions in numbers of adult oligodendrocytes and myelinated axons in several areas, including the ACC and CC.

Coupling of spindle oscillations in the prefrontal cortex and sharpwave-ripples in the hippocampus is thought to have a role in memory consolidation. The authors explored whether adult oligodendrogenesis influences this coupling. Mrf ${ }^{\mathrm{CKO}}$ and control mice were treated with $4 \mathrm{OHT}$ and then trained in a contextual fear conditioning task. The authors recorded neural activity in the ACC and CA1 of the mice before and after training. In the control mice, training led to an increase in the correlation of spindle and ripple amplitudes, but this increased coupling was not observed in the $M r f^{\mathrm{CKO}}$ mice. Moreover, these mice showed reduced freezing compared with controls after training. These findings suggest that adult oligodendrogenesis is important for learning-induced increases in coupling between the PFC and hippocampus.

Overall, these findings indicate that oligodendrogenesis and de novo myelination have a key role in memory consolidation in adult mice.

Darran Yates

ORIGINAL ARTICLE Steadman, P. E. et al. Disruption of oligodendrogenesis impairs memory consolidation in adult mice. Neuron https://doi.org/ 10.1016/j.neuron.2019.10.013 (2019) 